\title{
Time Synchronization of Automation Controllers for Power Applications
}

\author{
David C. Mazur \\ Member, IEEE \\ Rockwell Automation Inc. \\ 1201 South Second Street \\ Milwaukee, WI 53204, USA \\ dcmazur@ra.rockwell.com
}

\author{
Ryan D. Quint \\ Student Member, IEEE \\ Virginia Polytechnic Institute and SU \\ 302 Whittemore Hall \\ Blacksburg, VA 24060, USA \\ quintr@vt.edu
}

\author{
Virgilio A. Centeno \\ Senior Member, IEEE \\ Virginia Polytechnic Institute and SU \\ 302 Whittemore Hall \\ Blacksburg, VA 24060, USA \\ virgilio@vt.edu
}

\begin{abstract}
This paper investigates various forms of industrial time synchronization and attempts to apply them to Programmable Automation Controllers (PACs) for power system applications. PACs have been used in industry for many control applications, but advancements in synchronization technology has allowed for PACs to be used in high speed control and data acquisition systems. This paper looks at utilizing a PAC as a time synchronized Sequence of Events recorder (SER) for substation automation. It is shown that a PAC can be time synchronized to multiple time references and provide accuracies accepted by the power industry.
\end{abstract}

Index Terms-Global Positioning System (GPS), Inter-range Instrumentation Group (IRIG) time codes, Precision Time Protocol (PTP), Programmable Automation Controller (PAC), Programmable Logic Controller (PLC)

\section{INTRODUCTION}

The recent widespread use of GPS receivers and precision timing protocols for accurate timing application has made it possible to send data to the controller with a high precision time stamp timestamp. For power systems and other wide area systems, the ability to utilize this new high precision time synchronization not only extracts additional information from the available data but also enables the implementation of wide area monitoring and control operations. With this precise timing now available at the programmable automation controller (PAC) level, PACs can now be used in a variety of markets to implement high speed control and data acquisition systems. Time synchronization for power system applications can be expanded from the current applications of synchrophasor in transmission systems to sequence of events recording, data acquisition, and other protection applications in the substation. This paper explores various levels of time synchronization and how they were applied to currently available technology to create a sequence of events recorder.

\section{APPLICATIONS OF TIME SYNCHRONIZATION}

This section investigates examples of applications that require time synchronization. Take the example of having a local controller with a remote $\mathrm{I} / \mathrm{O}$ device. Currently, measurements are typically taken by controller-based I/O to ensure that accurate timestamp information of events is recorded. Due to advancement in time synchronization, this application can now be addressed by utilizing distributed I/O
[1]. A sensor now can be connected to a remote $\mathrm{I} / \mathrm{O}$ device. If each device contains a clock, and they are time synchronized, discrete transitions from off-to-on and on-to-off can be captured and timestamped as an input event. This timestamp now has meaning since both clocks are now synchronized to each other, and this information can be sent across the network and used by the controller or any other enterprise level process. Since the clocks are synchronized, the controller can use the information for sequence of events recording or timestamped data logging [2]. As a result, more than just traditional data can be sent across a time synchronized network. In addition to timestamped inputs, scheduled outputs and synchronized actuation can also be achieved over time synchronized networks. Four major areas of improvement can be realized with the implementation of time synchronized networks: sequence of events recording, timestamped data logging, coordinated/synchronized operation, and motion control.

For power system applications, time synchronization plays a major role in everyday process administration. Examples of time synchronization can be seen in Sequence of Events recording (SER), protection schemes, power quality measurements, and data acquisition. Microprocessor relays typically have the capability to synchronize to a common reference time source such as GPS through IRIG-B [3]. Relatively newer technology including wide-area measurement systems using synchronized phasor measurement units (PMUs) apply a GPS time stamp to realtime data frames to capture a snapshot of the power system. More traditionally, remote terminal units (RTUs) within a substation record events from devices throughout the station and report statuses to a SCADA (Supervisory Control and Data Acquisition) Master, commonly at a control center [4]. Although SCADA is not a real-time application due to its synchronous design, a valid timestamp of events from substation equipment via either an RTU or other intelligent electronic device is beneficial for both online and offline applications.

\section{LiterATURE REVIEW - TIMING PROTOCOLS}

It is important to note that when attempting to synchronize multiple devices over a distributed network, various timing protocols will provide different levels of synchronization accuracy. This section provides an overview of the four most 
prevalent timing protocols that exist in the industrial marketplace today. These protocols include: Global Positioning System (GPS), Inter-range Instrumentation Group (IRIG) time codes, Network Time Protocol (NTP), and Precision Time Protocol (PTP).

\section{A. Global Positioning System-GPS}

Global Positioning System (GPS) is not only a navigation system, but has evolved to become the world's primary mean of distributing precise time and frequency. GPS was developed by the United States Department of Defense (DoD) in 1978, and is still maintained by the organization today [5]. Currently, 31 satellites orbit the earth and provide accurate time within +/- $10 \mathrm{~ns}$ to GPS receivers [2]. The US DoD dictates the accuracy of the system, and has the right to limit this accuracy. Additionally, GPS systems can be costly to deploy over a distributed I/O network as a GPS receiver is required at each node, and the antenna must have a clear view of the sky to attain a locked signal.

\section{B. Inter-Range Intercommunications Group Time Codes}

In 1952, the commanders of the U.S. guided missile test ranges formed the Inter-Range Instrumentation (IRIG) group as part of the Range Commanders Council (RCC) of the U.S. Army in order to share information about range instrumentation [6]. Today, the Steering Committee and ten Technical Working Groups including the Telecommunications and Timing Group (TTG) control the IRIG time code standards. The IRIG standard was last updated in September, 2004 and is titled IRIG Serial Time Code Formats [7]. The most well-known and utilized is the IRIG-B time code from this standard. Despite multiple choices of IRIG code formats, IRIG-B is the mostly widely used in the power industry. In fact, more than ninety percent of substations use the IRIG-B format, which is typically better than $+/-1 \mathrm{~ms}$ [8].

There exist two different forms of the IRIG-B time code: modulated and un-modulated. Modulated IRIG-B is transmitted on a carrier frequency sinusoid (similar to AM radio), and as a result, must be demodulated at the receiving end in order to interpret the time data. With advancements in phase locked loop (PLL) technology, modulated IRIG-B schemes can obtain accuracies within +/- $10 \quad \mu \mathrm{s}$ [9]. Installations with modulated IRIG-B must be isolated through some form of transformer in order to prevent ground loops and signal degradation. Un-modulated IRIG-B, or level shifted IRIG, is the alternative to the traditional modulated approach. This form of time code transmission uses digital level shifting to achieve data transfer. As no demodulation is necessary at the receiving end, time synchronization accuracy improves to $+/-1 \mu \mathrm{S}$ [9]. The experimental setup described in this paper utilizes both modulated and un-modulated IRIG-B.

\section{Network Time Protocol-NTP}

Network time protocol (NTP) is a protocol for synchronizing clocks of computers using a data network, such as the intranet or a wide area network (WAN). This protocol was developed at the University of Delaware in 1980 and it is the first protocol to address time synchronization over variable latency packet switched networks [2]. This protocol provides accuracies that depend on the setup of the network between each device and the performance of the computers' operating systems [10]. Ideally, the connections in the network should be as short as possible, but this protocol does include methods to estimate and account for round-trip path delay. Overall, the accuracy of this protocol is in the low tens of milliseconds over wide area networks (WANs) and better than a millisecond over local area networks (LANs) [2].

\section{Precision Time Protocol-PTP}

The formal title of IEEE 1588 is Precision Clock Synchronization for Networked Measurement and Control Systems. This protocol is better known as Precision Time Protocol or PTP [11]. The standard specifies a protocol to synchronize independent clocks operating on separate nodes of a distributed measurement and control network to a high degree of accuracy and precision [2]. PTP is often used to synchronize distributed $\mathrm{I} / \mathrm{O}$ devices over variable latency packet switched networks such as Ethernet. This protocol was originally released in 2002 defining many of the regulations and specifications, and later revised to its current version in 2008 and released as IEEE 1588v2. The original version of this standard defined ordinary and boundary clocks while version 2 defines transparent and hybrid clocks; all of these clock types will be addressed in this section [11].

In its simplest form, PTP was intended to be an administration-free protocol. The clocks within a network will communicate with each other across a network media and establish multiple master-slave relationships. These masterslave relationships form what is known as a hierarchy of clocks [11]. The overall intent of PTP is that distributed I/O devices will manage the synchronization of clocks automatically, thus requiring little if any network administrator input. Not all devices within a network require the same level of time synchronization accuracy; as a result, PTP allows for the support of a wide spectrum of clock accuracies to support the needs of the end device or process [12]. For example, when dealing with protective relays, accuracy within a millisecond is acceptable due to mechanical component tolerances, but when dealing with PMUs, submicrosecond accuracy is needed to meet the IEEE C37.1182005 standard [13]. PTP can be configured to meet the needs of both of these applications. Additionally, PTP can use multiple sources of time as an ultimate time reference including, but not limited to, Global Positioning System (GPS), IRIG, Network Time Protocol (NTP), or another PTP clock. 


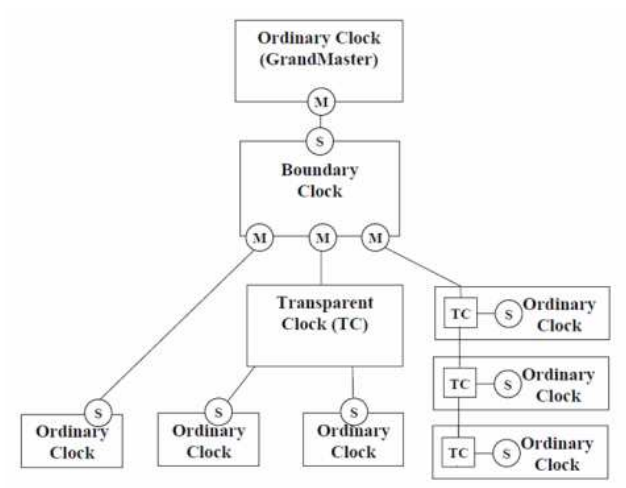

Fig. 1 Generic Network of Clocks

A system or network of clocks by definition will consist of one or more devices capable of becoming a master clock, while other devices within the network will serve as slave clocks [11]. Normally one master clock will be designated as the grandmaster clock.

Fig. 1 depicts the combinations of clocks that PTP supports: ordinary, boundary, transparent, and hybrid. Ordinary clocks consist of a single connection port, which industry commonly refers to as a PTP port. This port can either be assigned as a master or a slave [2]. Examples of ordinary clocks include GPS receivers and logic controllers and are typically located at the end nodes of a network [14]. Boundary clocks contain multiple PTP ports that establish separate PTP domains by segmenting the synchronization path between master and slave clocks. As their name implies, boundary clocks form boundaries between PTP synchronization segments [11]. These clocks are typically found in network switches. Transparent clocks are very different from ordinary and boundary clocks in that they help compensate for the propagation delay through the network rather than segment the network [2]. Finally, hybrid clocks are defined as a combination of PTP type clocks in a device. The most common type of hybrid clock is a transparent clock paired with an ordinary clock. Hybrid clocks are usually found within motion devices and used to perform synchronized actuation [2].

It was previously stated that PTP was designed to be an administration free protocol in that devices negotiate amongst themselves to determine a hierarchy of clocks. The algorithm used to determine the hierarchy of these clocks is known as the Best Master Clock Algorithm (BMCA). The BMCA, as defined by IEEE 1588 , is the strict arbitration process employed to determine the status of each network node, either master or slave [11]. As its name implies, the BMCA will determine the best master clock and name it the grandmaster of the PTP system. All remaining clocks within the system are ultimately synchronized to the grandmaster.

Announce messages are sent approximately every two seconds for any PTP device claiming to be a master. These announce messages contain information about how good the clock is compared to a scale [1]. When a node receives an announce message from another device it compares the credentials that it receives to its own. The better of the two clocks will serve as the master, while the lesser will act as the slave. This process continues until the status for every clock within the network is determined.

The BMCA utilizes four criteria to determine the better of two clocks. These factors include: clock class, accuracy, variance, and priority. Clock class defines the relative measure of clock quality. Accuracy defines how close the clock meters time to an absolute reference. Variance is the measure of the clock's stability. Priority is a manual override that can be established if a network administrator wants one clock to serve as grandmaster over another [11].

One main advantage to implementing PTP in a system is that it will dynamically update to topology changes [2]. For example, if the current grandmaster is removed from the system, the BMCA will attempt to designate a new grandmaster from the remaining clocks. On the other hand, if a clock with better credentials is added to the system, the BMCA will designate this new clock as grandmaster.

Fig. 2 depicts the synchronization process for clocks utilizing the PTP protocol. PTP utilizes four messages in order to synchronize two clocks: sync message, follow up message, delay request message, and delay response message.

These messages are transmitted from master to slave and allow the clocks to make frequency adjustments to adjust the rate at which the clocks meter time. Additionally, the messages allow for the clocks to measure the phase delay between master and slave and allow for a value correction. The frequency adjustment is made by utilizing the sync and follow up messages, while the phase adjustment is made by utilizing the delay request and delay response messages [2]. Every time that the synchronization process occurs, timestamps $\mathrm{t} 1 \mathrm{-t} 4$ are collected to determine the offset from master and frequency adjustments for the slave clocks. Clocks that are PTP compliant that make both a frequency and value adjustment are known as tunable clocks [2].

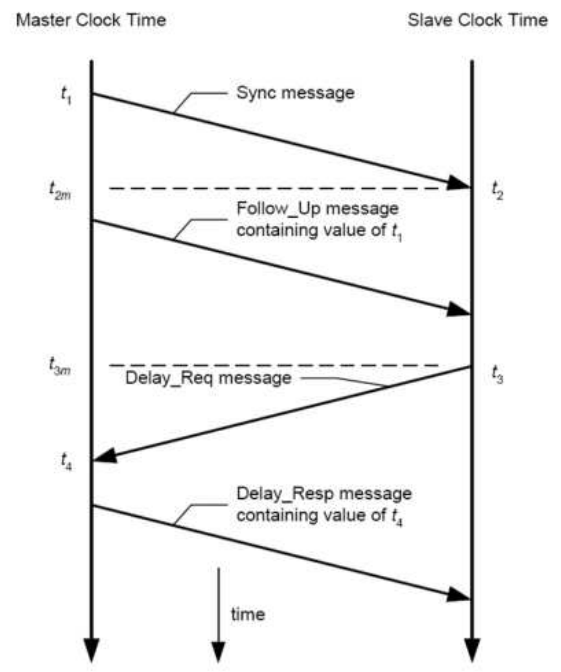

Fig. 2 Synchronization Messages 


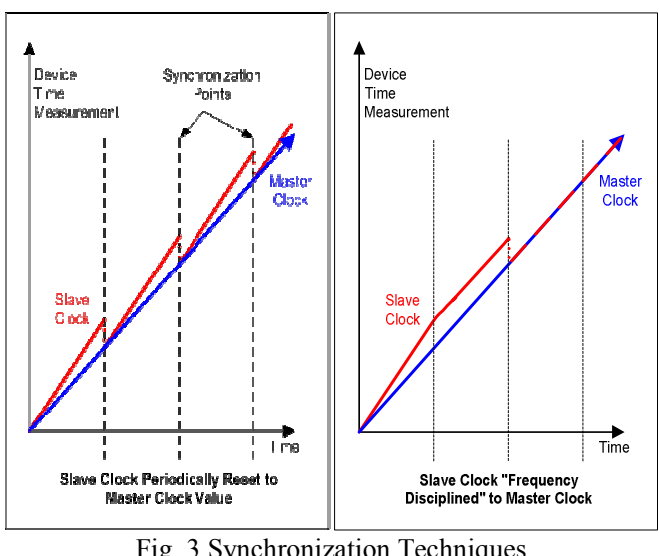

Tunable clocks are very important to use in any variable latency packet switched network. Take the example with two clocks, one master and one slave and start them at the exact same time. No two clocks will meter time at the exact same rate due to the natural frequencies of the individual clock crystals. Consequently, the clocks will begin to diverge from each other and no longer remain synchronized. One approach to correct this problem is to periodically reset the slave clock's value to that of the master's. This solution does not address the issue of differing clock frequencies or the rate at which the two clocks meter time. As a result, the clocks will only be brought into alignment for a moment in time, then begin to diverge again. Tunable clocks on the other hand, allow for proper synchronization of clocks. These clocks allow for the frequency of the slave clock to be tuned to that of its master so they will meter time at the same rate[2]. In addition to making a frequency adjustment, the tunable clock also applies a value or offset adjustment so that master and slave clocks are truly synchronized. Both synchronization phenomena can be seen in Fig. 3 .

PTP is only a time synchronization protocol and must be implemented on a network that supports the protocol. IEEE 1588 was designed for packet switched networks such as Ethernet. For experiments described later in this paper, the EtherNet/IP (EtherNet/Industrial Protocol) was implemented across the network. EtherNet/IP is a protocol that is part of the Common Industrial Protocol or CIP suite.

\section{E. Common Industrial Protocol - CIP}

The Common Industrial Protocol (CIP) is an industrial protocol suite that contains message and service instructions for automation applications pertaining to control, safety, synchronization, and motion [15]. This protocol is currently managed by the Open DeviceNet Vendors Association (ODVA) and is currently an open development network supported by hundreds of industrial vendors [16]. CIP allows for these applications to be implemented on enterprise-level Ethernet networks. Benefits of implementing CIP networks include: seamless integration of $\mathrm{I} / \mathrm{O}$ control and data collection, information flow across multiple networks, and implementable multilayer networks without the need for the implementation of network bridges [17].
CIP can be defined as an object-oriented connection based protocol that supports both explicit and implicit messaging. Explicit message connections provide generic, multipurpose communications paths between devices. These messages provide typical request/response type network communication [15]. Explicit messages are used by CIP for configurations, monitoring, and troubleshooting. Implicit messages, also known as I/O connections, provide special purpose communications paths between producing and consuming agents within a network. This $\mathrm{I} / \mathrm{O}$ data is often exchanged cyclically or at a requested packet interval (RPI) [15].

The principle behind how EtherNet/IP synchronizes time across a network of distributed $\mathrm{I} / \mathrm{O}$ can be attributed to the CIP Sync Object [1]. This object provides an interface to CIP Sync that allows devices such as logic controllers to access the synchronization mechanism. CIP Sync defines an offset clock model that addresses the requirements for various control applications. This model is necessary as PTP defines a mechanism for distributing and synchronizing time, but fails to define a mechanism for compensating for step changes in time that may occur at the grandmaster source [2].

Fig. 4 defines the CIP Sync Object at a high level. This model shows a PTP master represented by the circle and a PTP time slave represented by the top right side rectangle. In this example PTP is used to discipline a local clock such that it ticks and meters time at the same rate of the PTP master. The slave clock also maintains an offset between the local clock time and the PTP system time. Any small delta, or step change in time, will cause the slave device to make a small adjustment to the "system to local clock offset" value [1]. In addition, the slave device will continue to tune its clock. A large step change will result in the device updating the offset value, but not tune its clock. As a result, cyclic tasks such as SCADA reads, can be scheduled based upon the local clock and will not be affected by large step changes in time at the grandmaster source.

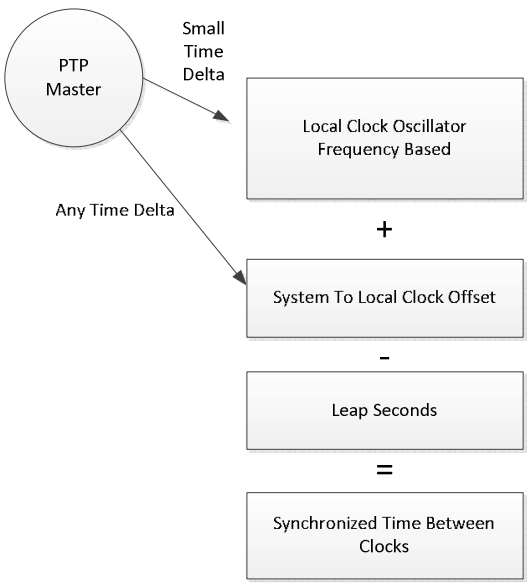

Fig. 4 CIP Sync Object Model

CIP Sync represents time as a 64 bit long-integer (LINT) that can be expressed in either nanoseconds or microseconds. The starting reference point in time for CIP Sync is January 1, 1970 starting at 12:00 AM. This time is represented in 
Universal Time Coordinated (UTC) and adjusted to include leap seconds [1]. In order to represent this 64 bit LINT in a readable format, an algorithm must be developed to compute the current date and time in a readable, understandable format for analysis. The algorithm consisted of several mathematical operations to convert the LINT into two strings that are in the following format: MM/DD/YYYY and HH:MM:SS: $\mu \mathrm{S} \mu \mathrm{S} \mu \mathrm{S} \mu \mathrm{S}$. The remainder of this paper will discuss an implementation of a Sequence of Events (SOE) recorder implemented by utilizing PTP in order to time synchronize events.

\section{SYSTEM ARCHITECTURE}

To test the interoperability and integration of the different synchronization protocols discussed in this paper, a laboratory setup was constructed utilizing a PAC as a time synchronized sequence of events (SOE) recorder. This section describes how the PAC and other devices were set up and configured to act as a time synchronized network. GPS time is obtained from a GPS antenna and distributed to multiple devices via IRIG-B protocols. The PAC is synchronized to UTC using a precision timing module IRIG$\mathrm{B}$ input time. Configured as the grandmaster clock, other modules within the PAC network are synchronized via PTP. Industrial components used in this setup include a programmable automation controller (PAC), managed switch, satellite-synchronized GPS clock, and three intelligent electronic devices (IEDs) set as instantaneous overcurrent $(50 \mathrm{P} 1)$ relays.

Fig. 5 illustrates an overview of the configuration for the sequence of events recorder system constructed. This system can be separated into two areas: a hardwired section and a packet switched network section. The hardwired section consists of relays monitoring phase currents in real time comparing these measurements to their $50 \mathrm{P} 1$ settings. The normally open auxiliary contacts on the relays are electrically wired to the PAC. These connections are used to send the tripping outputs (52A) from the relays to the PAC. Upon transition from $0 \rightarrow 24 \mathrm{VDC}$ input on the PAC SOE module, a time stamp is applied to the measurements. This is discussed in detail in the following sections.

The packet switched network section of the overall system contains three primary components: a programming remote terminal, managed switch, and PAC. This section is configured in a star network topology with the managed switch acting as the central node in the star. Distribution of timing, communication between the programming terminal and the PAC, and collection and monitoring of results were performed in this section.

\section{A. Programmable Automation Controller}

The solution chosen for implementation of the SOE recorder was a rack-based automation controller that accepts multiple modules with various configurations [18]. This platform operates asynchronously, meaning it utilizes two

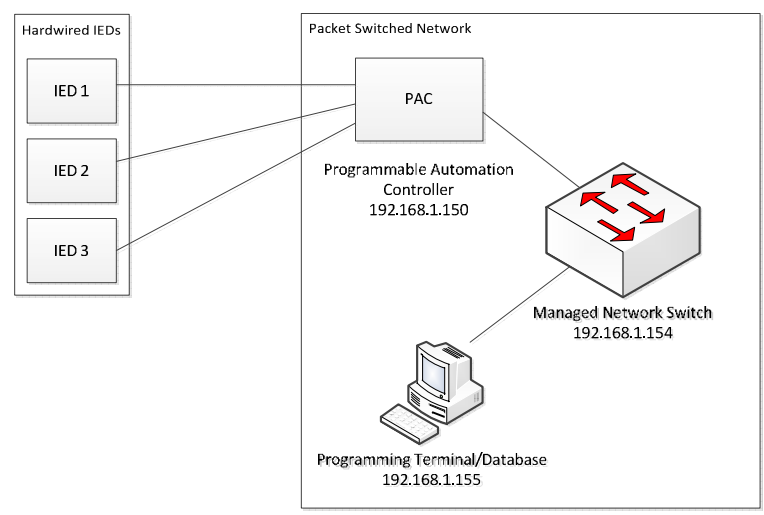

Fig. 5 System Configuration

sets of memory. These banks of memory are the program and $\mathrm{I} / \mathrm{O}$ sets and are stored separately and can be written to independently. This means that $\mathrm{I} / \mathrm{O}$ information can be collected separately from the executing instructions in program memory [18]. I/O memory is populated via the use of a backplane circuit and a Requested Packet Interval (RPI). Multiple data words can be simultaneously shared between modules connected to the backplane as well as written to $\mathrm{I} / \mathrm{O}$ mapped memory locations [19]. For use as a high-speed, high-accuracy timing device in power system protection, monitoring, and control, asynchronous operation is beneficial. The relay inputs to the SOE module occur at different times, and accurate recording of these times must occur immediately rather than on the next scan through the program. The processor chosen is capable of true multitasking which allows for asynchronous gathering of information and simultaneous synchronization to UTC time while normally schedule programs continue to execute.

TABLE I

Programmable Automation Controller HaRdware

\begin{tabular}{|c|c|c|}
\hline Slot & Part Number & Description \\
\hline $\mathbf{0}$ & $1756-\mathrm{L} 73$ & Processor \\
\hline $\mathbf{1}$ & $1756-\mathrm{IB} 16 \mathrm{SOE}$ & $15-30$ VDC SOE Module \\
\hline $\mathbf{2}$ & $1756 \mathrm{HP}-\mathrm{TIME}$ & Precision Timing Module \\
\hline $\mathbf{3}$ & $1756-\mathrm{EN} 2 \mathrm{~T}$ & EtherNet/IP Bridge \\
\hline
\end{tabular}

Table I defines the layout for the PAC used in the SOE recorder constructed. The processor chosen for this project contained a duo-core Intel chipset that operates as a RISC machine [20]. This module is where programs are written, downloaded, and executed. The Sequence of Events (SOE) module is seated in Slot 1 of the PAC. The SOE module accepts 12-30 VDC discrete signals from external devices. When the input circuit is toggled from either $\mathrm{OFF} / \mathrm{ON}$ or ON/OFF, the module applies a 64-bit timestamp to the event. The EtherNet/IP Bridge allows the PAC to communicate with other network devices through the managed switch. The precision timing module allows for the PAC to be time synchronized to a specified source. Synchronizing the PAC to an external time reference, rather than its local time, is critical for comparison of event times across a network of devices, as discussed below. 


\section{B. Time Synchronization}

A sequence of events recorder has three major requirements for use in a distributed environment:

1. The SOE recorder must provide time stamps in a timely manner, such that the time an event occurs can be recorded accurately.

2. For the record to provide useful time to a system operator, the time stamps must be synchronized to a known reference clock time.

3. When multiple devices are synchronized to the same reference (either through identical or different protocols, i.e. GPS through IRIG-B or distributed through PTP), the SOE must also be referenced to that time for comparison between the devices and the SOE recorder.

To address the first requirement, the PAC SOE module is capable of recording time stamps for both rising and falling edges of input measurements. The 64-bit timestamp is configured in the SOE module designed to only record rising edge inputs, which correspond to triggering events from the relays. The second requirement is met by using the precision timing module to provide UTC time. This module accepts multiple forms of timing inputs including GPS, IRIG-B.122, Network Time Protocol (NTP), and PTP [21]. Additionally, the module synchronizes the PAC and the rest of the packet switched network by providing a PTP output. The precision timing module acts as a grandmaster clock with highest priority while the other modules, specifically the SOE module, act as slaves. Distribution of this time is accurate to $+/-100 \mathrm{~ns}$ of the master source [2]. The timing module contains a two port switch that allows for the distribution of PTP to the rest of the network [22]. One port of the timing module switch is directly connected to the managed switch, which connects to the remote programming unit and serves as an additional distribution point of PTP through the network. Table II defines the PTP hierarchy of clocks for the SOE recorder system constructed.

At each rising edge input to the SOE module, a time stamp is recorded corresponding to that input signal. The time stamp applied to this event is based on the time observed by the SOE module. Since the SOE module is time synchronized to the precision timing module output via PTP,

TABLE II

PTP CLOCK DEFINITIONS

\begin{tabular}{|c|c|c|}
\hline Device & Clock Type & IP Address \\
\hline Timing Module & Grandmaster & 192.168 .1 .250 \\
\hline EtherNet/IP Bridge & Boundary & 192.168 .1 .150 \\
\hline Managed Switch & Transparent & 192.168 .1 .154 \\
\hline L73 Processor & Ordinary & N/A \\
\hline Remote Terminal & Ordinary & 192.168 .1 .155 \\
\hline
\end{tabular}

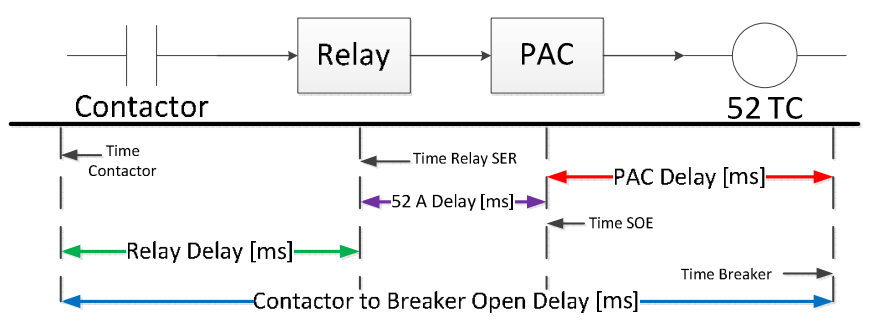

Fig. 6 Timing Delays

the SOE timestamps are also synchronized to UTC time. The accuracy of the timestamp applied to the SOE input signals is sub-millisecond $[12,23]$. A GPS antenna gathers UTC time, which is accurate to $+/-10 \mathrm{~ns}$, as mentioned above. This time is received at a satellite-synchronized clock commonly used in substation applications. The accuracy of this device is +/$500 \mathrm{~ns}$ and $+/-1 \mu \mathrm{s}$ for un-modulated and modulated IRIG-B, respectively [24]. The time acquired by the GPS clock is distributed to the relays and PAC through IRIG-B. The difference between the two distributions of time is the type of IRIG used. The relays are configured to accept IRIG-B.000 which is a un-modulated format, while the PAC timing module is configured to accept modulated IRIG-B.122 [21].

The third requirement is important for systems where comparison of times across a network may be required. In some situations, this may not be an issue. Take for example the simple case of closing a contactor and recording its time of closure. The output of the PAC to energize the trip coil can be timestamped using the PAC SOE module. This corresponds to the time that the PAC initiates the event. The auxiliary contact acts as an input and is recorded and is also timestamped at the PAC SOE module. The difference between these times is the time required for the signal to be observed at the trip coil, the contactor to close, and the auxiliary contact to close and enable the SOE input.

The SOE recorder implementation can also act as a delay monitor and recorder for offline analysis. Using a network reference to UTC time, all timestamps applied to measurements can be compared against each other regardless of where they occur in the system. Intelligent electronic devices (IEDs) generally have the capability to apply a timestamp to I/O. As mentioned, the relays are synchronized to GPS via IRIG-B.000 while the PAC is synchronized to IRIG-B.122 due to hardware constraints.

This feature allows for an estimate of delay for any signal within the system. Fig. 6 shows the time synchronization system architecture with measured delays labeled. The following equations describe how each delay is calculated:

$$
\begin{aligned}
T_{R 1} & =T_{R 1, S E R}-T_{S O E, \text { contactor }} \\
T_{R 2} & =T_{R 2, S E R}-T_{S O E, \text { contactor }} \\
T_{R 3} & =T_{R 3, S E R}-T_{S O E, \text { contactor }} \\
T_{R 1-S O E} & =T_{S O E, R 1}-T_{S O E, \text { contactor }} \\
T_{R 2-S O E} & =T_{S O E, R 2}-T_{S O E, \text { contactor }} \\
T_{R 3-S O E} & =T_{S O E, R 3}-T_{S O E, \text { contactor }} \\
T_{P A C}=T_{S O E, P A C \_O} \text { Out } & -\left(T_{S O E, R 1}\left\|T_{S O E, R 2}\right\| T_{S O E, R 3}\right)
\end{aligned}
$$


For example, let us explore a single tripping event and the measurements recorded. Refer back to Fig. 6 for the diagram and (1) for the calculation of these delays. The contactor is energized via a PAC AC output module. The time that the auxiliary contact is closed is considered the baseline measurement of time for the rest of the test. It is assumed that the contactor along with its auxiliary contact close simultaneously as all poles are mechanically interlocked [25]. The input step change is observed at the relays and they determine whether to energize their output contacts based on their individual pickup level.

\section{TEST PROCEDURE AND RESULTS}

A test setup was constructed to test the efficacy of the time synchronized PAC acting as a sequence of events recorder for relay tripping scenarios. A simple transmission model circuit was configured such that upon energizing a contactor initiated by the PAC, the relays would all receive current measurements. These measurements were compared against the overcurrent elements (50P1) programmed into the IEDs. In addition, the 50P1 settings were varied to simulate different tripping situations.

As mentioned, each relay was synchronized via IRIGB.000 through a BNC connector to a GPS clock. Additionally, the PAC was also synchronized to the GPS clock, but using IRIG-B.122 based on the requirement of the timing module. To test whether the relays and the PAC were synchronized to the same reference, tripping events were recorded at both the relays and the PAC. A timing table was set up in the PAC such that a rising edge input to the SOE module would overwrite the previous time recorded in the table. The relays are able to store up to 20 records viewable from a remote terminal but store up to 1000 records that can be exported as a *.txt file at the remote terminal [26]. Using the latter method, tripping sequences were performed and the results were extracted for comparison with PAC times.

The PAC and relay times were considered precise once the difference between the event times was within manufacturer specifications for contact closure. Functionality of the PAC as a sequence of events recorder was then explored. For all tripping scenarios tested, the PAC properly identified the incoming trip signal and applied a UTC time stamp to the measurement using SOE model and precision timing module time.

TABLE I

SYNCHRONIZATION ACCURACIES

\begin{tabular}{|c|c|}
\hline Point of Synchronization & Accuracy \\
\hline GPS Antenna to Receiver & $+/-10 \mathrm{~ns}$ \\
\hline GPS Clock (modulated) & $+/-1 \mu \mathrm{s}$ \\
\hline IRIG-B.122 & $+/-10 \mu \mathrm{s}$ \\
\hline IEEE 1588 PTP Protocol & $+/-100 \mathrm{~ns}$ \\
\hline PAC Sequence of Events Module & $+/-10 \mu \mathrm{s}$ \\
\hline Total Accuracy of PAC Synchronization & $+/-21 \boldsymbol{\mu s}$ \\
\hline
\end{tabular}

The viability of the PAC as a sequence of events recorder synchronized to a wide-area reference time is dependent on the accuracy of timing achievable. Accuracy for each synchronization method was collected from the literature. Table III shows the accuracies for the different points of synchronization. As the time synchronization propagates from the GPS satellites to the PAC SOE module, each subsection is accurate to a given value. The worst case scenario of PAC synchronization accuracy is the summation of all component accuracies.

In addition to the PAC utilized as an SOE recorder, delays in the protection scheme were quantified using the PAC to record all assertion times of the various measurement points in the system. Fig. 7 and Fig. 8 show results for testing delays within the system with three sigma error bars. As the figure shows, latency between the contactor closing and the relays recording a tripping event were between 3.009-8.114 ms. The SOE module recorded trip times, and the latency between relay energizing its contacts and SOE input enabling was 3.679-5.039 ms. The PAC was set to send a digital status via DC output signal following any single relay input signal, which allowed for PAC delay computation. This latency was measured as $1.8 \mathrm{~ms}$ maximum.

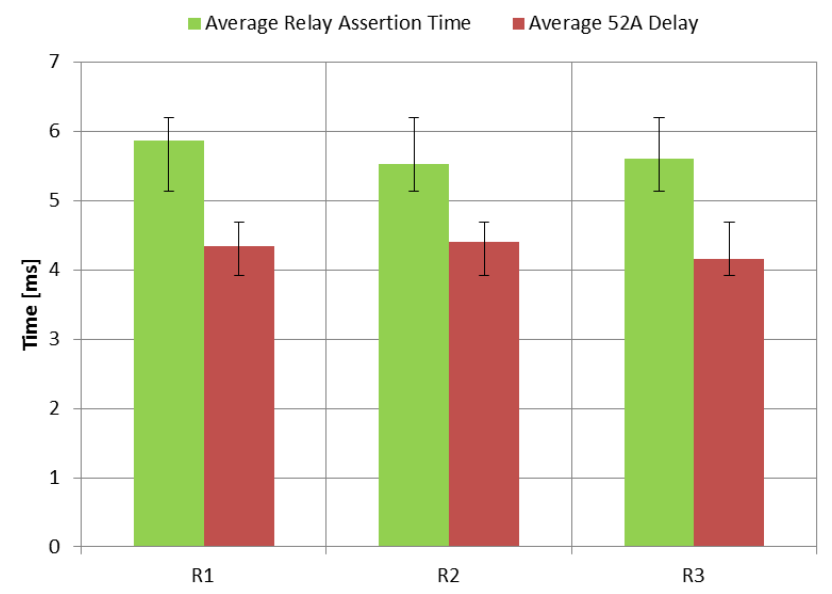

Fig. 7. System Delays

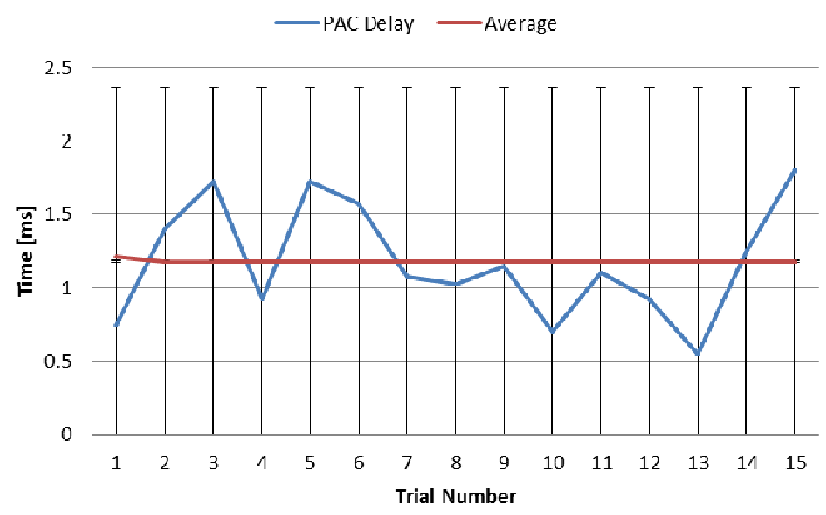

Fig. 8. Automation Controller Delays 


\section{CONCLUSIONS}

PACs have evolved into highly capable devices able to perform high-speed computation, network between various protocols, and synchronize to external time references. These benefits are explored and the efficacy of a PAC in power system applications, especially timing and monitoring, is illustrated. A SOE recorder system using a PAC to perform time stamping and data collection is evaluated.

PACs have an advantage in that they their solutions are customizable based on the modules used and the design considerations taken. For this system, a precision timing module was included to synchronize the PAC to UTC through a GPS clock distributing IRIG-B. This time was further distributed over a packet switch network via IEEE 1588 PTP protocol, using timing module as the grandmaster clock. By synchronizing to UTC, the PAC timestamps provided were comparable to the relay sequential events records (SERs).

Results show that the PAC performs efficiently and effectively as a sequential events recorder for both real-time and offline analyses. Protection system delays were evaluated by collecting data for offline analysis. PAC maximum delays for processing $\mathrm{I} / \mathrm{O}$ and outputting corresponding digital signals were $1.8 \mathrm{~ms}$. In addition, due to the synchronicity of all components in the system overall system delays were analyzed, with maximum relay SER latency of $8.114 \mathrm{~ms}$ and SOE input latency of $5.039 \mathrm{~ms}$.

PAC synchronization accuracy was determined to be approximately $+/-21 \mu$ s using the designed configuration and synchronization methods. This is well-suited for a substation environment, considering a power system $60 \mathrm{~Hz}$ cycle requires $16.667 \mathrm{~ms}$. The interoperability of a PAC, in conjunction to its timely synchronization and customizability, make it a viable and effective addition to current power system applications.

\section{REFERENCES}

[1] R. A. Inc. (2010). Integrated Architecture and CIP Sync Configureation.

[2] R. A. Inc., "Precision Time Protocol Over EtherNet/IP," in Motion Over EtherNet/IP Learning Series, R. A. Inc., Ed., ed. Milwaukee, WI: Rockwell Automation Inc., 2011.

[3] R. C. Council, "IRIG Serial Time Codes Formats," ed: Range Commanders Council Telecommunications and Timing Group, 2004.

[4] I. PES, "IEEE Standard for SCADA and Automation Systems," vol. IEEE Std C37.1-2007 (Revision of IEEE Std C37.1-1994), ed, 8 May 2008.

[5] L. M. N. M. A. Lombardi, A. N. Novick, V. S. Zhang, "Time and Frequency Measurements Using the Global Positioning System," Cal. Lab. Int. J. Metrology, pp. 26-33, 2001.

[6] B. W. Pike, "IRIG, Inter-Range Instrumentation Group----History, Functions and Status, 1959," Space Electronics and Telemetry, IRE Transactions on, vol. SET-6, pp. 59-61, 1960.

[7] "IRIG Serial Time Code Formats," in IRIG Standard 200-04 ed. U.S. Army White Sands Missle Range, New Mexico: Range Commanders Council, Sept. 2004.

[8] B. Dickerson, "Time in the Power Industry: How and Why We Use It," Arbiter Systems, Inc.

[9] I. P. PSRC, "IRIG-B Time Code Connection Requirements."

[10] D. L. Mills, "Internet time synchronization: the network time protocol," Communications, IEEE Transactions on, vol. 39, pp. 1482-1493, 1991.
[11] "IEEE Standard for a Precision Clock Synchronization Protocol for Networked Measurement and Control Systems," IEEE Std 1588-2008 (Revision of IEEE Std 1588-2002), pp. c1-269, 2008.

[12] Cisco, "CIP Sync Sequence of Events," in Converged Plantwide Ethernet (CPwE) Design and Implementation Guide, Cisco, Ed., ed, 2011, p. 72.

[13] "IEEE Standard for Synchrophasors for Power Systems," vol. IEEE Std C37.118-2005 (Revision of IEEE Std 1344-1995), ed, 2005.

[14] S. Hekmat. (January 2011, Communication Networks. 24-39.

[15] L. M. Surhone, M. T. Tennoe, and S. F. Henssonow, Common Industrial Protocol: VDM Verlag Dr. Mueller AG \& Co. Kg, 2010.

[16] ODVA, "The Common Industrial Protocol (CIP) and the Family of CIP Networks," ODVA, Ed., ed, 2010, p. 92.

[17] ODVA. (2011). ODVA EtherNet/IP. Available: http://www.odva.org/Home/ODVATECHNOLOGIES/EtherNetIP/tab $\mathrm{id} / 67 /$ lng/en-US/language/en-US/Default.aspx

[18] R. A. Inc., "Control Logix System," R. A. Inc., Ed., ed. Milwaukee, WI: Rockwell Automation Inc., 2011, p. 44.

[19] R. A. Inc., "Integrated Architecture Technical Resources," R. Automation, Ed., ed. Milwaukee, WI: Rockwell Automation Inc., 2010 , p. 60.

[20] R. A. Inc. (2011). 1756-UM001L-EN-P-May-2011.

[21] R. A. Inc., "EtherNet/IP Basics," 2011.

[22] H. T. R. Automation. (2011). 1756 HP Time Version 2 User Manual. [23] R. A. Inc. (2008). 1756-UM528A-EN-P.

[24] Schweitzer Engineering Laboratories. (2011). SEL-2407 SatelliteSynchronized Clock Instruction Manual.

[25] R. A. Inc. (2004). 100C , 4 Pole Contactors Available: http://iterature.rockwellautomation.com/idc/groups/literature/docum ents/pp/100-pp025_-en-p.pdf

[26] Schweitzer Engineering Laboratories. (2011). SEL-421 Relay Protection and Automation System Instruction Manual.

David C. Mazur was born in Pittsburgh, PA in 1989. He received his Bachelor of Science in Electrical Engineering summa cum laude from Virginia Polytechnic Institute and State University, Blacksburg, VA in May 2011. David graduated first in class and his studies focused on power systems and process control. David graduated with his Master of Science degree in May 2012 for his work based on rotor angle measurement and speed governor control of synchronous machines. He plans to graduate with his Ph.D. in May of 2014. His research interests include electric machinery and control, time synchronization networks, substation automation, and intelligent agent electrical distribution schemes.

David currently works for Rockwell Automation, Milwaukee, WI, as a research and development engineer. His recent focus has been on the development of regenerative variable frequency drive technology. David has also helped develop technology for time synchronized continuous casters for the steel industry. He also runs a project that aims to assess the power quality of AsiaPacific and European countries.

Ryan D. Quint was born in Palo Alto, CA in 1988. He received his Bachelor of Science in electrical engineering from the University of Washington in 2010. He received his Master of Science in electrical engineering from the Department of Electrical and Computer Engineering, Virginia Polytechnic Institute and State University in December 2011 and is currently pursuing the Ph.D. degree. His research interests include wide area measurement systems, synchrophasor-based protection and control, data latency in power systems, and cascading events.

Since June 2009, Ryan has worked at the Bonneville Power Administration, Portland, OR. His industry experience has included generation interconnections, phasor measurement unit testing, design of remedial action schemes, and synchrophasor-based control of power systems.

Virgilio A. Centeno (M'92-SM'06) received the M.S. and Ph.D. degrees in electrical engineering from Virginia Polytechnic Institute and State University (Virginia Tech), Blacksburg, in 1988 and 1995, respectively.

He worked as a Project Engineer at Macrodyne, Inc., Clifton Park, NY, in the development of phasor measurement units from 1991 to1997. He joined the faculty of Virginia Tech as a Visiting Professor in the fall of 1997and became an Associate Professor in 2007. His area of interest is wide area measurement and its applications to power system protection and control. 